\title{
Goeken-Johnson Sixth-Order Runge-Kutta Method
}

\author{
Mohammed M. Ismail \\ Department of Computer Sciences \\ College of Computer and Mathematical Sciences \\ University of Mosul
}

Received
$03 / 01 / 2010$
Accepted

05 / 05 / 2010

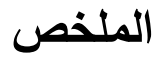

تم في هذا البحث اشتقاق طريقة رن ج-كوتا الجديدة من الرتبة السادسة اعتماداً على طريقة

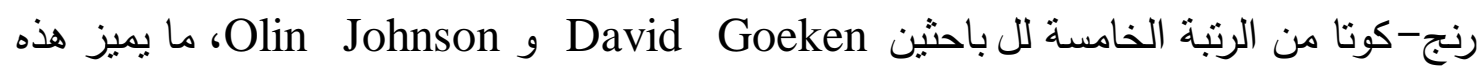
الطريقة أنها نتطلب خمسة تقييمات لـ f فقط، في حين أن الطريقة الاعتيادية تتطلب ستة أو أو سبعة تقييمات لـ f ، بعد ذلك ثتم مقارنة هذه الطريقة بطريقة رنج-كوتا الجديدة من الرتبة الخامسة.
\end{abstract}

\begin{abstract}
In this paper we drive a new sixth-order Runge-Kutta method, depending on the new fifth order Runge-Kutta method of David Goeken and Olin Johnson, the property of this method is that it needs five function evaluations only where the standard method needs six or seven function evaluations, then this method is compared with the new fifth order RungeKutta method.
\end{abstract}

\section{1- Introduction:-}

Given $y^{\prime}=f(y)$, standard Runge-Kutta methods perform multiple evaluations of $\mathrm{f}(\mathrm{y})$ in each integration sub-interval as required for a given accuracy. Evaluations of $\mathrm{y}^{\prime \prime}=\mathrm{f}_{\mathrm{y}}(\mathrm{x}, \mathrm{y}) \mathrm{f}(\mathrm{x}, \mathrm{y})$ or higher derivatives are not considered due to the assumption that the calculations involved in these functions exceed those of $\mathrm{f}$. However, $\mathrm{y}^{\prime \prime}$ can be approximated to sufficient accuracy from past and current evaluations of $f$ to achieve a higher order of accuracy than is available through current functional evaluations alone.

In July of 1998 at the ANODE (Auckland Numerical Ordinary Differential Equations) Workshop, the two scientists David Goeken and Olin Johnson introduced a new class of Runge-Kutta methods based on this observation [4],[11]. They presented a third order method which requires 
only two evaluations of $\mathrm{f}$ and a fourth-order method which requires three and fifth order method which requires four. This paper reviews the fifthorder methods and gives the general solution to the equations generated by the sixth-order methods of this new class. Interestingly, these sixth-order methods require only five functional evaluations per step whereas standard Runge-Kutta methods require six or seven.

\section{2- Third-order method:-}

Goeken and Johnson consider initial value problems expressed in autonomous form. Starting with the non-autonomous form, they assume that $f(x, y)$ is a continuous function with domain $D$ in $\operatorname{IR}^{n+1}$ where $x \in R, y$ $\in \mathrm{IR}^{\mathrm{n}}$ and $(\mathrm{x}, \mathrm{y}) \in \mathrm{D}$. They assume that:- [10], [12]

$$
\left\|f\left(x, y_{1}\right)-f\left(x, y_{2}\right)\right\|_{2} \leq \mathrm{L}\left\|y_{1}-y_{2}\right\|_{2}
$$

for all $\left(x, y_{1}\right),\left(x, y_{2}\right) \in D$ (where $L$ is a Lipschitz constant); thus the problem

$$
\begin{gathered}
\mathrm{y}^{\prime}=\mathrm{f}(\mathrm{x}, \mathrm{y}) \\
\mathrm{y}\left(\mathrm{x}_{0}\right)=\mathrm{y}_{0} \text { with }\left(\mathrm{x}_{0}, \mathrm{y}_{0}\right) \in \mathrm{D}
\end{gathered}
$$

has a unique solution.

In autonomous form, $\mathrm{y}$ and $\mathrm{f}$ have $\mathrm{n}+1$ components with $y_{n+1}=f_{n+1}(x, y)=x$ and $f_{n+1}(y)=1$. The initial value problem is then written:

$$
\begin{aligned}
& y^{\prime}=f(y) \\
& y\left(x_{0}\right)=y_{0} \text { where }\left(y_{0}\right)_{n+1}=f_{n+1}\left(x_{0}, y_{0}\right)=x_{0}
\end{aligned}
$$

Most efforts to increase the order of the Runge-Kutta methods have been accomplished by increasing the number of Taylor's series terms used and thus the number of functional evaluations required [10],[5],[12] and [9]. The use of higher order derivative terms has been proposed for stiff problems [16] and [7]. The method adds higher order derivative terms to the Runge-Kutta $k_{\mathrm{i}}$ terms ( $\mathrm{i}>1$ ) to achieve a higher order of accuracy, For more details see [3] [6]. For example, the new third-order method, GJ3, for autonomous systems, is:- [10]

$$
\mathrm{y}_{\mathrm{n}+1}=\mathrm{y}_{\mathrm{n}}+\mathrm{b}_{1} \mathrm{k}_{1}+\mathrm{b}_{2} \mathrm{k}_{2}
$$

and $\mathrm{k}_{1}=\mathrm{hf}\left(\mathrm{y}_{\mathrm{n}}\right)$. However, they introduce additional terms by assigning:[4], [10],[12].

$$
\mathrm{k}_{2}=\mathrm{hf}\left(\mathrm{y}_{\mathrm{n}}+\mathrm{a}_{21} \mathrm{k}_{1}+\mathrm{ha}_{22} \mathrm{f}_{\mathrm{y}}\left(\mathrm{y}_{\mathrm{n}}\right) \mathrm{k}_{1}\right)
$$

Using Taylor's series expansion techniques, the above is uniquely satisfied to $\mathrm{O}\left(\mathrm{h}^{3}\right)$ as follows:

\section{3- Fourth-order method:-}

$$
\begin{aligned}
& \mathrm{k}_{1}=\operatorname{hf}\left(\mathrm{y}_{\mathrm{n}}\right) \\
& \mathrm{k}_{2}=\operatorname{hf}\left(\mathrm{y}_{\mathrm{n}}+\frac{2}{3} \mathrm{k}_{1}+\frac{2}{9} \mathrm{hf}_{\mathrm{y}}\left(\mathrm{y}_{\mathrm{n}}\right) \mathrm{k}_{1}\right) \\
& \mathrm{y}_{\mathrm{n}+1}=\mathrm{y}_{\mathrm{n}}+\frac{1}{4} \mathrm{k}_{1}+\frac{3}{4} \mathrm{k}_{2}
\end{aligned}
$$


Similarly, the fourth-order method, GJ4, for autonomous systems, is:[10],[12]

and

$$
\mathrm{y}_{\mathrm{n}+1}=\mathrm{y}_{\mathrm{n}}+\mathrm{b}_{1} \mathrm{k}_{1}+\mathrm{b}_{2} \mathrm{k}_{2}+\mathrm{b}_{3} \mathrm{k}_{3}
$$

$$
\begin{aligned}
& k_{1}=\operatorname{hf}\left(y_{n}\right) \\
& k_{2}=h f\left(y_{n}+a_{21} k_{1}+h a_{22} f_{y}\left(y_{n}\right) k_{1}\right) \\
& k_{3}=h f\left(y_{n}+a_{31} k_{1}+a_{32} k_{2}+h a_{33} f_{y}\left(y_{n}\right) k_{1}+h a_{34} f_{y}\left(y_{n}\right) k_{2}\right)
\end{aligned}
$$

The Taylor's series expansion of these higher order methods is tedious and error prone. Goeken and Johnson used modern symbolic mathematical packages to expand and then to solve the resulting systems of nonlinear equations that were generated. In this work, they used the symbolic mathematical packages [14],[10], [11], and [7].

The general solution to the system of equations (with $\mathrm{a}_{34}=0$ ) has been found with example solutions are shown in:

and

$$
\mathrm{y}_{\mathrm{n}+1}=\mathrm{y}_{\mathrm{n}}+\frac{1}{6} \mathrm{k}_{1}+\frac{1}{6} \mathrm{k}_{2}+\frac{2}{3} \mathrm{k}_{3}
$$

$$
\begin{aligned}
k_{1} & =\operatorname{hf}\left(\mathrm{y}_{\mathrm{n}}\right) \\
\mathrm{k}_{2} & =\operatorname{hf}\left(\mathrm{y}_{\mathrm{n}}+\mathrm{k}_{1}+\frac{1}{2} \mathrm{hf}_{\mathrm{y}}\left(\mathrm{y}_{\mathrm{n}}\right) \mathrm{k}_{1}\right) \\
\mathrm{k}_{3} & =\operatorname{hf}\left(\mathrm{y}_{\mathrm{n}}+\frac{3}{8} \mathrm{k}_{1}+\frac{1}{8} \mathrm{k}_{2}\right)
\end{aligned}
$$

\section{4- Fifth order method:-}

In July of 1998, Goeken and Johnson presented [11] this numerical integration technique at a meeting attended by John Butcher. Using his tree-based approach [5], Butcher suggested a fifth-order method. Since the meeting, his technique has been verified using Taylor's series expansion techniques to determine the general solution for the fifth-order methods. The fifth-order method, GJ5, for autonomous systems, is:- [10],[12]

$$
\mathrm{y}_{\mathrm{n}+1}=\mathrm{y}_{\mathrm{n}}+\mathrm{b}_{1} \mathrm{k}_{1}+\mathrm{b}_{2} \mathrm{k}_{2}+\mathrm{b}_{3} \mathrm{k}_{3}+\mathrm{b}_{4} \mathrm{k}_{4}
$$

and

$$
\begin{aligned}
& \mathrm{k}_{1}=\operatorname{hf}\left(\mathrm{y}_{\mathrm{n}}\right) \\
& \mathrm{k}_{2}=\operatorname{hf}\left(\mathrm{y}_{\mathrm{n}}+\mathrm{a}_{21} \mathrm{k}_{1}+\mathrm{ha}_{22} \mathrm{f}_{\mathrm{y}}\left(\mathrm{y}_{\mathrm{n}}\right) \mathrm{k}_{1}\right) \\
& \mathrm{k}_{3}=\operatorname{hf}\left(\mathrm{y}_{\mathrm{n}}+\mathrm{a}_{31} \mathrm{k}_{1}+\mathrm{a}_{32} \mathrm{k}_{2}+\mathrm{ha}_{33} \mathrm{f}_{\mathrm{y}}\left(\mathrm{y}_{\mathrm{n}}\right) \mathrm{k}_{1}\right) \\
& \mathrm{k}_{4}=\operatorname{hf}\left(\mathrm{y}_{\mathrm{n}}+\mathrm{a}_{41} \mathrm{k}_{1}+\mathrm{a}_{42} \mathrm{k}_{2}+\mathrm{a}_{43} \mathrm{k}_{3}+\mathrm{ha}_{44} \mathrm{f}_{\mathrm{y}}\left(\mathrm{y}_{\mathrm{n}}\right) \mathrm{k}_{1}\right)
\end{aligned}
$$

The solution presented by Butcher and verified using the above system of equations is:

and

$$
\begin{aligned}
& \mathrm{k}_{1}=\operatorname{hf}\left(\mathrm{y}_{\mathrm{n}}\right) \\
& \mathrm{k}_{2}=\operatorname{hf}\left(\mathrm{y}_{\mathrm{n}}+\frac{1}{3} \mathrm{k}_{1}+\frac{1}{18} \mathrm{hf}_{\mathrm{y}}\left(\mathrm{y}_{\mathrm{n}}\right) \mathrm{k}_{1}\right) \\
& \mathrm{k}_{3}=\operatorname{hf}\left(\mathrm{y}_{\mathrm{n}}-\frac{152}{125} \mathrm{k}_{1}+\frac{252}{125} \mathrm{k}_{2}-\frac{44}{125} \mathrm{hf}_{\mathrm{y}}\left(\mathrm{y}_{\mathrm{n}}\right) \mathrm{k}_{1}\right) \\
& \mathrm{k}_{4}=\operatorname{hf}\left(\mathrm{y}_{\mathrm{n}}+\frac{19}{2} \mathrm{k}_{1}-\frac{72}{7} \mathrm{k}_{2}+\frac{25}{14} \mathrm{k}_{3}+\frac{5}{2} \mathrm{hf}_{\mathrm{y}}\left(\mathrm{y}_{\mathrm{n}}\right) \mathrm{k}_{1}\right)
\end{aligned}
$$




$$
\mathrm{y}_{\mathrm{n}+1}=\mathrm{y}_{\mathrm{n}}+\frac{5}{48} \mathrm{k}_{1}+\frac{27}{56} \mathrm{k}_{2}+\frac{125}{336} \mathrm{k}_{3}+\frac{1}{24} \mathrm{k}_{4}
$$

\section{5- Sixth order method:-}

We drive now the Sixth order, GJ6, for autonomous systems, lets:

$$
\mathrm{y}_{\mathrm{n}+1}=\mathrm{y}_{\mathrm{n}}+\mathrm{b}_{1} \mathrm{k}_{1}+\mathrm{b}_{2} \mathrm{k}_{2}+\mathrm{b}_{3} \mathrm{k}_{3}+\mathrm{b}_{4} \mathrm{k}_{4}+\mathrm{b}_{5} \mathrm{k}_{5}
$$

and

$$
\begin{aligned}
& \mathrm{k}_{1}=\operatorname{hf}\left(\mathrm{y}_{\mathrm{n}}\right) \\
& \mathrm{k}_{2}=\operatorname{hf}\left(\mathrm{y}_{\mathrm{n}}+\mathrm{a}_{21} \mathrm{k}_{1}+h \mathrm{~h}_{22} \mathrm{f}_{\mathrm{y}}\left(\mathrm{y}_{\mathrm{n}}\right) \mathrm{k}_{1}\right) \\
& \mathrm{k}_{3}=\operatorname{hf}\left(\mathrm{y}_{\mathrm{n}}+\mathrm{a}_{31} \mathrm{k}_{1}+\mathrm{a}_{32} \mathrm{k}_{2}+h \mathrm{ha}_{33} \mathrm{f}_{\mathrm{y}}\left(\mathrm{y}_{\mathrm{n}}\right) \mathrm{k}_{1}\right) \\
& \mathrm{k}_{4}=\operatorname{hf}\left(\mathrm{y}_{\mathrm{n}}+\mathrm{a}_{41} \mathrm{k}_{1}+\mathrm{a}_{42} \mathrm{k}_{2}+\mathrm{a}_{43} \mathrm{k}_{3}+h \mathrm{ha}_{44} \mathrm{f}_{\mathrm{y}}\left(\mathrm{y}_{\mathrm{n}}\right) \mathrm{k}_{1}\right) \\
& \mathrm{k}_{5}=\operatorname{hf}\left(\mathrm{y}_{\mathrm{n}}+\mathrm{a}_{51} \mathrm{k}_{1}+\mathrm{a}_{52} \mathrm{k}_{2}+\mathrm{a}_{53} \mathrm{k}_{3}+\mathrm{a}_{54} \mathrm{k}_{4}+h \mathrm{~h}_{55} \mathrm{f}_{\mathrm{y}}\left(\mathrm{y}_{\mathrm{n}}\right) \mathrm{k}_{1}\right)
\end{aligned}
$$

The sixth order Goeken-Johnson can be able to generate the Taylor's series expansion of the above, and we get the following systems of equations:- [10]

$$
\begin{aligned}
& b_{1}+b_{2}+b_{3}+b_{4}+b_{5}=1 \\
& b_{2} a_{21}+b_{3}\left(a_{31}+a_{32}\right)+b_{4}\left(a_{41}+a_{42}+a_{43}\right)+b_{5}\left(a_{51}+a_{52}+a_{53}+a_{54}\right)=\frac{1}{2} \\
& b_{2} a_{21}{ }^{2}+b_{3}\left(a_{31}+a_{32}\right)^{2}+b_{4}\left(a_{41}+a_{42}+a_{43}\right)^{2}+b_{5}\left(a_{51}+a_{52}+a_{53}+a_{54}\right)^{2}=\frac{1}{3} \\
& b_{2} a_{21}{ }^{3}+b_{3}\left(a_{31}+a_{32}\right)^{3}+b_{4}\left(a_{41}+a_{42}+a_{43}\right)^{3}+b_{5}\left(a_{51}+a_{52}+a_{53}+a_{54}\right)^{3}=\frac{1}{4} \\
& b_{2} a_{21}{ }^{4}+b_{3}\left(a_{31}+a_{32}\right)^{4}+b_{4}\left(a_{41}+a_{42}+a_{43}\right)^{4}+b_{5}\left(a_{51}+a_{52}+a_{53}+a_{54}\right)^{4}=\frac{1}{5} \\
& b_{2} a_{21}{ }^{5}+b_{3}\left(a_{31}+a_{32}\right)^{5}+b_{4}\left(a_{41}+a_{42}+a_{43}\right)^{5}+b_{5}\left(a_{51}+a_{52}+a_{53}+a_{54}\right)^{5}=\frac{1}{6} \\
& b_{3} a_{22} a_{32}+b_{4}\left(a_{21} a_{32} a_{43}+a_{22} a_{42}+a_{33} a_{43}\right)+b_{5}\left(a_{21} a_{32} a_{43} a_{54}+a_{22} a_{52}+a_{33} a_{53}+a_{44} a_{54}\right)=\frac{1}{24} \\
& b_{4} a_{21} a_{32} a_{43}+b_{5} a_{21} a_{32} a_{43} a_{54}=\frac{1}{120} \\
& b_{5} a_{21} a_{32} a_{43} a_{54}=\frac{1}{720} \\
& a_{21}+a_{22}=\frac{1}{2} \\
& a_{31}+a_{32}+a_{33}=\frac{1}{2} \\
& a_{41}+a_{42}+a_{43}+a_{44}=1 \\
& a_{51}+a_{52}+a_{53}+a_{54}+a_{55}=1 \\
& a_{10}
\end{aligned}
$$

Now we can form the sixth order formula (with $a_{21}=\frac{1}{4}$ ) we get:- [10] 


$$
\begin{aligned}
& \mathrm{a}_{22}=\frac{1}{4}, \mathrm{a}_{31}=\frac{-1}{4}, \mathrm{a}_{32}=\frac{1}{2}, \mathrm{a}_{33}=\frac{1}{4}, \mathrm{a}_{41}=\frac{1}{4}, \mathrm{a}_{42}=\frac{1}{4}, \mathrm{a}_{43}=\frac{1}{4}, \mathrm{a}_{44}=\frac{1}{4}, \\
& \mathrm{a}_{51}=\frac{-1}{4}, \mathrm{a}_{52}=\frac{1}{2}, \mathrm{a}_{53}=\frac{1}{4}, \mathrm{a}_{54}=\frac{1}{4}, \mathrm{a}_{55}=\frac{1}{4} \\
& \mathrm{~b}_{5} \mathrm{a}_{21} \mathrm{a}_{32} \mathrm{a}_{43} \mathrm{a}_{54}=\frac{1}{720} \Rightarrow \mathrm{b}_{5}\left(\frac{1}{4}\right)\left(\frac{1}{2}\right)\left(\frac{1}{4}\right)\left(\frac{1}{4}\right)=\frac{1}{720} \Rightarrow \frac{1}{128} \mathrm{~b}_{5}=\frac{1}{720} \Rightarrow \mathrm{b}_{5}=\frac{8}{45} \\
& \mathrm{~b}_{4} \mathrm{a}_{21} \mathrm{a}_{32} \mathrm{a}_{43}+\mathrm{b}_{5} \mathrm{a}_{21} \mathrm{a}_{32} \mathrm{a}_{43} \mathrm{a}_{54}=\frac{1}{120} \Rightarrow \frac{1}{32} \mathrm{~b}_{4}+\frac{8}{45}\left(\frac{1}{4}\right)\left(\frac{1}{2}\right)\left(\frac{1}{4}\right)\left(\frac{1}{4}\right)=\frac{1}{120} \\
& \frac{1}{32} b_{4}+\frac{1}{720}=\frac{1}{120} \Rightarrow b_{4}+\frac{4}{90}=\frac{4}{15} \Rightarrow b_{4}=\frac{4}{18} \\
& b_{3} a_{22} a_{32}+b_{4}\left(a_{21} a_{32} a_{43}+a_{22} a_{42}+a_{33} a_{43}\right)+b_{5}\left(a_{21} a_{32} a_{43} a_{54}+a_{22} a_{52}+a_{33} a_{53}+a_{44} a_{54}\right)=\frac{1}{24} \\
& \frac{1}{8} b_{3}+\frac{4}{18}\left(\frac{1}{32}+\frac{1}{16}+\frac{1}{16}\right)+\frac{8}{45}\left(\frac{1}{128}+\frac{1}{8}+\frac{1}{16}+\frac{1}{16}\right)=\frac{1}{24} \\
& \frac{1}{16} b_{3}+\frac{5}{144}+\frac{33}{720}=\frac{1}{24} \Rightarrow b_{3}+\frac{5}{9}+\frac{33}{45}=\frac{4}{6} \Rightarrow b_{3}+\frac{58}{45}=\frac{2}{3} \Rightarrow b_{3}=\frac{-28}{45} \\
& \mathrm{~b}_{2} \mathrm{a}_{21}+\mathrm{b}_{3}\left(\mathrm{a}_{31}+\mathrm{a}_{32}\right)+\mathrm{b}_{4}\left(\mathrm{a}_{41}+\mathrm{a}_{42}+\mathrm{a}_{43}\right)+\mathrm{b}_{5}\left(\mathrm{a}_{51}+\mathrm{a}_{52}+\mathrm{a}_{53}+\mathrm{a}_{54}\right)=\frac{1}{2} \\
& \frac{1}{4} b_{2}-\frac{28}{45}\left(\frac{-1}{4}+\frac{1}{2}\right)+\frac{4}{18}\left(\frac{1}{4}+\frac{1}{4}+\frac{1}{4}\right)+\frac{8}{45}\left(\frac{-1}{4}+\frac{1}{2}+\frac{1}{4}+\frac{1}{4}\right)=\frac{1}{2} \\
& \frac{1}{4} b_{2}-\frac{7}{45}+\frac{1}{6}+\frac{2}{15}=\frac{1}{2} \Rightarrow \frac{1}{2} b_{2}-\frac{14}{45}+\frac{2}{6}+\frac{4}{15}=1 \Rightarrow \frac{1}{2} b_{2}+\frac{13}{45}=1 \Rightarrow b_{2}=\frac{16}{45} \\
& b_{1}+b_{2}+b_{3}+b_{4}+b_{5}=1 \Rightarrow b_{1}+\frac{16}{45}-\frac{28}{45}+\frac{4}{18}+\frac{8}{45}=1 \Rightarrow b_{1}+\frac{12}{90}=1 \Rightarrow b_{1}=\frac{78}{90}
\end{aligned}
$$

By using the above systems of equations we get the following systems of general solution:

$$
\mathrm{y}_{\mathrm{n}+1}=\mathrm{y}_{\mathrm{n}}+\frac{78}{90} \mathrm{k}_{1}+\frac{16}{45} \mathrm{k}_{2}-\frac{28}{45} \mathrm{k}_{3}+\frac{4}{18} \mathrm{k}_{4}+\frac{8}{45} \mathrm{k}_{5}
$$

and

$$
\begin{aligned}
& \mathrm{k}_{1}=\operatorname{hf}\left(\mathrm{y}_{\mathrm{n}}\right) \\
& \mathrm{k}_{2}=\operatorname{hf}\left(\mathrm{y}_{\mathrm{n}}+\frac{1}{4} \mathrm{k}_{1}+\frac{1}{4} \mathrm{hf}_{\mathrm{y}}\left(\mathrm{y}_{\mathrm{n}}\right) \mathrm{k}_{1}\right) \\
& \mathrm{k}_{3}=\operatorname{hf}\left(\mathrm{y}_{\mathrm{n}}-\frac{1}{4} \mathrm{k}_{1}+\frac{1}{2} \mathrm{k}_{2}+\frac{1}{4} \mathrm{hf}_{\mathrm{y}}\left(\mathrm{y}_{\mathrm{n}}\right) \mathrm{k}_{1}\right) \\
& \mathrm{k}_{4}=\operatorname{hf}\left(\mathrm{y}_{\mathrm{n}}+\frac{1}{4} \mathrm{k}_{1}+\frac{1}{4} \mathrm{k}_{2}+\frac{1}{4} \mathrm{k}_{3}+\frac{1}{4} \mathrm{hf}_{\mathrm{y}}\left(\mathrm{y}_{\mathrm{n}}\right) \mathrm{k}_{1}\right) \\
& \mathrm{k}_{5}=\operatorname{hf}\left(\mathrm{y}_{\mathrm{n}}-\frac{1}{4} \mathrm{k}_{1}+\frac{1}{2} \mathrm{k}_{2}+\frac{1}{4} \mathrm{k}_{3}+\frac{1}{4} \mathrm{k}_{4}+\frac{1}{4} \mathrm{hf}_{\mathrm{y}}\left(\mathrm{y}_{\mathrm{n}}\right) \mathrm{k}_{1}\right)
\end{aligned}
$$

As we know that the standard sixth order method has 6 or 7 $\mathrm{k}_{\mathrm{i}}$ 's function evaluations but in this method the $\mathrm{k}_{\mathrm{i}}$ 's function evaluations are 5 only. 


\section{6- Stability Region of Sixth order method:-}

The stability properties of numerical methods of Ordinary Differential equations determined by study the behavior of these methods, by using test problem:- [1],[2],[13]

$$
\mathrm{y}^{\prime}=\lambda \mathrm{y}, \mathrm{y}\left(\mathrm{x}_{0}\right)=\mathrm{y}_{0}
$$

Where $\lambda$ is real, the real solution of the above problem when $x=x_{n}$ is:

$$
\mathrm{y}\left(\mathrm{x}_{\mathrm{n}+1}\right)=\mathrm{e}^{\lambda \mathrm{h}} \mathrm{y}\left(\mathrm{x}_{\mathrm{n}}\right)
$$

This implies the use of the general formula of Runge-Kutta method:[1],[2],[13]

Where:

$$
\mathrm{y}_{\mathrm{n}+1}=\mathrm{y}_{\mathrm{n}}+\mathrm{h} \phi\left(\mathrm{y}_{\mathrm{n}}, \mathrm{h}\right)
$$

$$
\begin{aligned}
& \phi\left(\mathrm{y}_{\mathrm{n}}, \mathrm{h}\right)=\sum_{\mathrm{r}=1}^{\mathrm{R}} \mathrm{b}_{\mathrm{r}} \mathrm{k}_{\mathrm{r}} \\
& \mathrm{k}_{\mathrm{r}}=\mathrm{f}\left(\mathrm{y}_{\mathrm{n}}+\mathrm{h} \sum_{\mathrm{s}=1}^{\mathrm{R}} \mathrm{a}_{\mathrm{rs}} \mathrm{k}_{\mathrm{s}}\right), \mathrm{r}=1,2, \ldots \ldots ., \mathrm{R} \\
& \mathrm{b}_{\mathrm{r}}=\sum_{\mathrm{s}=1}^{\mathrm{R}} \mathrm{a}_{\mathrm{rs}}, \mathrm{r}=1,2, \ldots \ldots \ldots \ldots . \mathrm{R}
\end{aligned}
$$

By using test problem we get the difference equation:- [1],[2],[13]

$$
\mathrm{y}_{\mathrm{n}+1}=\mathrm{E}(\lambda \mathrm{h}) \mathrm{y}_{\mathrm{n}}, \mathrm{n}=0,1,2,
$$

Where $E(\lambda \mathrm{h})$ is the stability function and converges to $\mathrm{e}^{\lambda \mathrm{h}}$ and $\mathrm{E}(\lambda \mathrm{h})<1$, by using equation (1) in the difference equation we get:[1],[2],[13]

$$
y\left(x_{n+1}\right)-y_{n+1}=O\left(h^{p+1}\right)
$$

Now by using test problem we get:- [1],[2]

$$
E(\bar{h})=r=1+\bar{h}+\frac{\bar{h}^{2}}{2 !}+\frac{\bar{h}^{3}}{3 !}+\ldots \ldots \ldots \ldots \ldots . .+\frac{\bar{h}^{p}}{p !}+O\left(\bar{h}^{p+1}\right)
$$

Where $\bar{h}=\lambda \mathrm{h}$, by using Taylor series we get:- [1],[2],[13]

$$
\mathrm{r}=\exp (\overline{\mathrm{h}})+\mathrm{O}\left(\overline{\mathrm{h}}^{\mathrm{p}+1}\right)
$$

Where $r$ is polynomial of $R$ stage in $\bar{h}$, if the method of stage $R$ then it is of rank $p$, therefore, $R \geq p$. Then we get:- [1],[2],[13]

$$
\mathrm{r}=1+\overline{\mathrm{h}}+\frac{\overline{\mathrm{h}}^{2}}{2 !}+\frac{\overline{\mathrm{h}}^{3}}{3 !}+\ldots \ldots \ldots \ldots \ldots . .+\frac{\overline{\mathrm{h}}^{\mathrm{p}}}{\mathrm{p} !}
$$

Then from Goeken-Johnson sixth order Runge-Kutta method $r$ is:

$$
\mathrm{r}=1+\overline{\mathrm{h}}+\frac{\overline{\mathrm{h}}^{2}}{2 !}+\frac{\overline{\mathrm{h}}^{3}}{3 !}+\frac{\overline{\mathrm{h}}^{4}}{4 !}+\frac{\overline{\mathrm{h}}^{5}}{5 !}
$$

and the stability region of Goeken-Johnson sixth order Runge-Kutta method is $(-3.2,0)$, we got the region by plotting $r$ in Figure(1). 


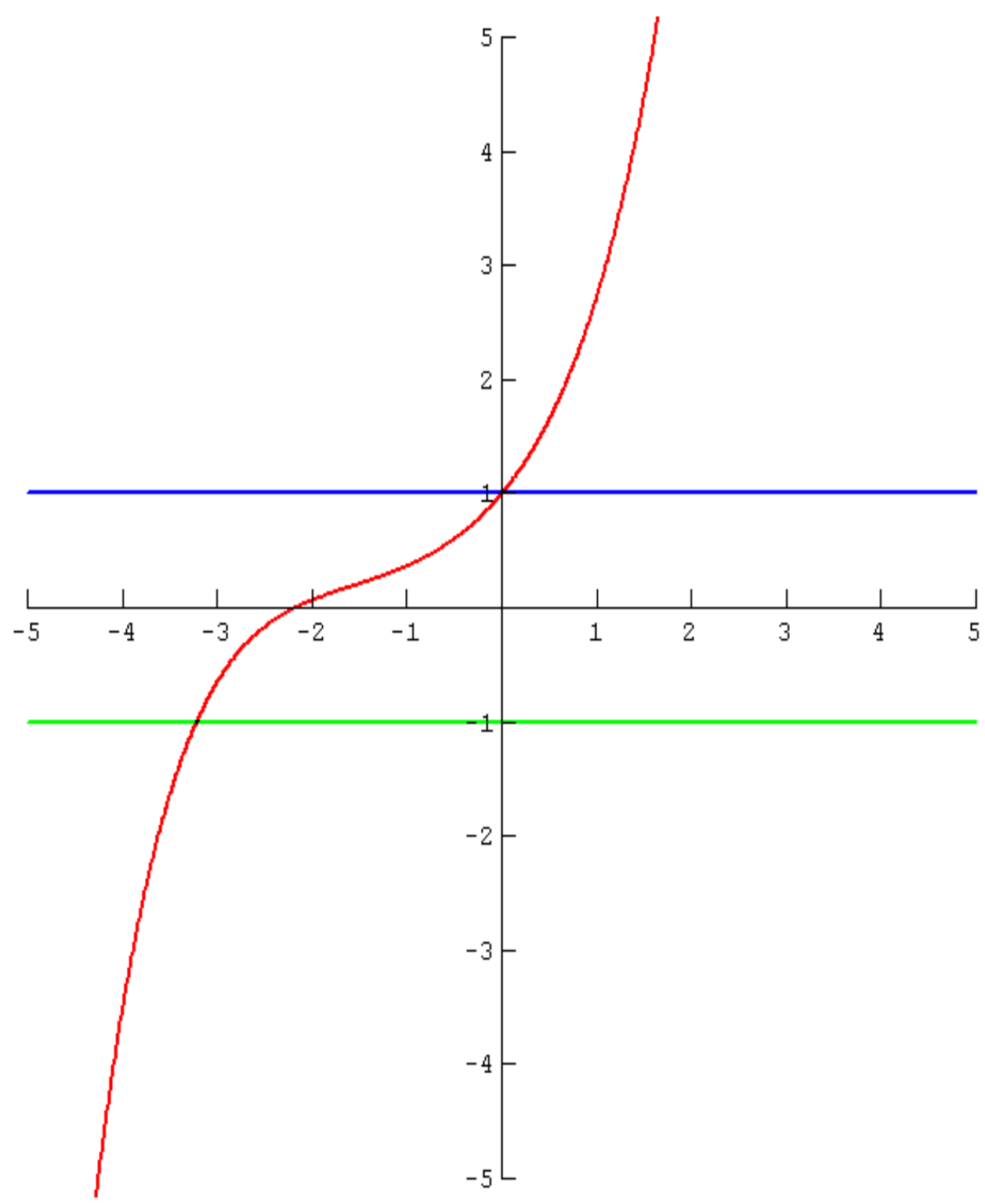

Figure(1): Stability region of Goeken-Johnson sixth order Runge-Kutta method

\section{7- Comparison:-}

In this section we compare with the fifth-order method, GJ5, and the sixth-order method, GJ6, and we get the solutions of the two methods have the same results, and the GJ6 is useful for solving any problems of Ordinary Differential equations, In this comparison we use the example $\mathrm{y}^{\prime}=-\mathrm{y}, \mathrm{y}(0)=1$, See table(1) and Figure(2) (The Figure is plotted in Matlab). 


\begin{tabular}{|c|c|c|c|c|}
\hline $\mathbf{x}$ & $\begin{array}{l}\text { Theoretical } \\
\text { solution }\end{array}$ & $\begin{array}{c}\text { Numerical solution } \\
\text { of GJ5 }\end{array}$ & $\begin{array}{c}\text { Numerical solution } \\
\text { of GJ6 }\end{array}$ & $\begin{array}{c}\text { Errors } \\
\text { (GJ5-GJ6) }\end{array}$ \\
\hline 0 & 1.0 & 1.0 & 1.0 & 0 \\
\hline 0.002 & 0.998001998667333 & 0.998001998600667 & 0.998000799822222 & $1.198778444400084 \mathrm{e}-006$ \\
\hline 0.004 & 0.996007989343991 & 0.996007989210925 & 0.996005596445795 & $2.392765129810570 \mathrm{e}-006$ \\
\hline 0.006 & 0.994017964053935 & 0.994017963854734 & 0.994014381880313 & 3.581974421296152e-006 \\
\hline 0.008 & 0.992031914837061 & 0.992031914571990 & 0.992027148151344 & $4.766420645840874 \mathrm{e}-006$ \\
\hline 0.01 & 0.990049833749168 & 0.990049833418492 & 0.990043887300400 & $5.946118092237107 \mathrm{e}-006$ \\
\hline 0.012 & 0.988071712861931 & 0.988071712465912 & 0.988064591384901 & 7.121081011085551e-006 \\
\hline 0.014 & 986097544262862 & 0.9860975438 & 0.986089252 & -006 \\
\hline 0.016 & 0.984127320055285 & 0.984127319529368 & 0.984117862669289 & $9.456860079026797 \mathrm{e}-006$ \\
\hline 0.018 & 0.982161032358301 & 0.982161031767827 & 0.982150414063287 & $1.061770453991695 \mathrm{e}-005$ \\
\hline 0.02 & 0.980198673306755 & 0.980198672651984 & 0.980186898780887 & $1.177387109696504 \mathrm{e}-005$ \\
\hline 0.022 & 0 & 0.9782402 & 0.978227308 & 5011011000 \\
\hline 0.024 & 0.976285709757909 & 0.976285708975320 & 0.976271636748612 & $1.407222670857244 \mathrm{e}-005$ \\
\hline 0.026 & 0.974335089608749 & 0.974335088762638 & 0.974319874318864 & $1.521444377405867 \mathrm{e}-005$ \\
\hline 0.028 & 0.972388366801247 & 0.972388365891871 & 0.972372013852914 & $1.635203895744564 \mathrm{e}-005$ \\
\hline 0.03 & 0.970445533548508 & 0.970445532576124 & 0.970428047549953 & $1.748502617093806 \mathrm{e}-005$ \\
\hline 0.032 & 0.968506582079198 & 0.968506581044060 & 0.968487967624770 & $1.861341928943716 \mathrm{e}-005$ \\
\hline 0.034 & 0.966571504637507 & 0.966571503539870 & 0.966551766307719 & $1.973723215098477 \mathrm{e}-005$ \\
\hline 0.036 & 0.964640293483123 & 0.964640292323242 & 0.964619435844685 & $2.085647855643025 \mathrm{e}-005$ \\
\hline 0.038 & 0.962712940891200 & 0.962712939669327 & 0.962690968497057 & $2.197117226987455 \mathrm{e}-005$ \\
\hline 0.04 & 0.960789439152323 & 0.960789437868711 & 0.960766356541692 & $2.308132701867027 \mathrm{e}-005$ \\
\hline 0.042 & 0.958869780572485 & 0.958869779227385 & 0.958845592270891 & $2.418695649331060 \mathrm{e}-005$ \\
\hline 0.044 & 0.956953957473047 & 0.956953956066710 & 0.956928667992362 & $2.528807434798441 \mathrm{e}-005$ \\
\hline 0.046 & 0.955041962190715 & 0.955041960723391 & 0.955015576029191 & $2.638469420013223 \mathrm{e}-005$ \\
\hline 0.048 & 0.953133787077505 & 0.953133785549444 & 0.953106308719813 & $2.747682963089027 \mathrm{e}-005$ \\
\hline 0.05 & 0.951229424500714 & 0.951229422912164 & 0.951200858417979 & 2.856449418497942e-005 \\
\hline
\end{tabular}

Table(1): Comparison between GJ5 and GJ6 


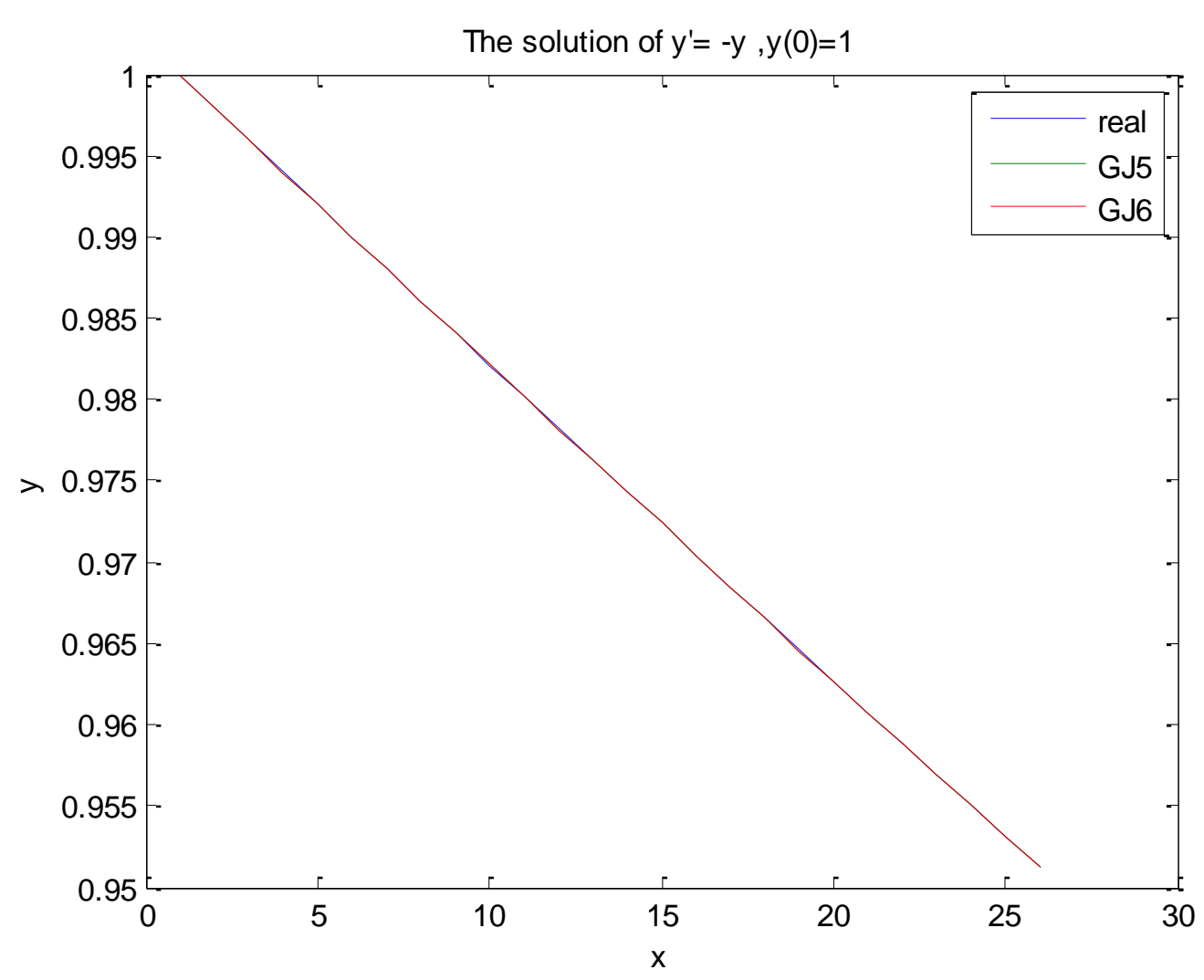

Figure(2): the Solution of $y^{\prime}=-y, y(0)=1$

\section{8- Conclusions:-}

New third-, fourth-, fifth-, and sixth-order numerical integration techniques inspired by the Runge-Kutta method have been presented. The new methods exploit the use of higher order derivatives, specifically $f_{y}$. In particular, a technique utilizing an approximation to $\mathrm{y}^{\prime \prime}$ has been presented resulting in a multistep Runge-Kutta method. The proposed methods are more efficient than the standard Runge-Kutta methods for cases:[4],[10],[12]

1. $f_{y}$ or $y^{\prime \prime}$ is cheaper to evaluate than $\mathrm{f}$.

2. The use of historical values of $f$ is cheaper than evaluating $f$,because the number of functional evaluations of standard Runge-Kutta methods are from 4 to 6 or 7 while the number of functional evaluations of new Runge-Kutta methods are from 2 to 5 only .

\section{References}

1) Abdullah, Ghanim M. S. (1999) Expand stability regions for some numerical methods, M.Sc thesis, Mosul university.

2) Abdul-Baky, Suhaib Abdul-Gabbar, (2002) Modified methods for solving Linear problems, M.Sc thesis, Mosul university. 
3) Butcher, J. C. (1996) A history of Runge-Kutta methods, Applied Numerical Mathematics 20, 247-260.

4) Butcher, John Chan, Robert Goodwin, Nicolette Grove, Bev and Heard, Allison (1998), ANODE: Auckland Numerical Ordinary Differential Equations. Department of Mathematics, The University of Auckland, 29 June-10 July 1998.

5) Butcher, J. C. (1987) The Numerical Analysis of Ordinary Differential Equations Runge-Kutta and General Linear Methods, John Wiley \& Sons Ltd., New York.

6) Butcher, J. C. and Wanner, G. (1996) Runge-Kutta methods: some historical notes, Applied Numerical Mathematics 22, 113-151.

7) Eaton, J. W. (1997) GNU Octave A high-level interactive language for numerical computations, http://www.we.fhosnabrueck.de/labsim/octave/manual/octave.html .

8) Enright, W. H. (1974) Second Derivative Multistep Methods for Stiff Ordinary Differential Equations, SIAM J. Numer. Anal., 11, 321331.

9) Gear, C. W. (1971) Numerical Initial Value Problems in Ordinary Differential Equations, Prentice-Hall, Englewood Cliffs, New Jersey.

10) Goeken, D. and Johnson, O. (1999) Fifth-order Runge-Kutta with Higher order Derivative Approxmations, $15^{\text {th }}$ Annual Conference of Applied Mathematics, Univ. of Central Oklahoma, Electronic Journal of Differential Equations, Conference 02, 1-9.

11) Goeken, D. and Johnson, O. (1999) Runge-Kutta with Higher Order Derivative Approximations, submitted to Applied Numerical Mathematics.

12) Kalaf, Bashir M. and Abdullah, Ghanim M. S. (2007) Modifying Runge-Kutta Methods With Higher Order Derivative Approximations, Journal of Education and Science, Vol. 19, No.2.

13) Kalaf, Bashir M. and Ismail, Mohammed M. (2008) Runge-Kutta methods of high order for solving stiff problems, Al-Rafiden Journal of Computer Sciences and Mathematics, Vol. 5, No.2.

14) PARI/GP (1999) http://hasse.mathematik.tu-muenchen.de/ntsw/pari/Welcome .

15) Reduce (1999) http://www.sub.uni-goettingen.de/ssg /math/infodata/000838.html .

16) Rosenbrock, H. H. (1963) Some General Implicit Processes for the Numerical Solution of Differential Equations, Comp. J., 5, 329-330. 